\title{
Outcome of Dengue infection in pregnancy at a tertiary care hospital in India
}

\author{
Naimah Raza ${ }^{1 *}$, Nitin P. Dhungat ${ }^{2}$

\begin{abstract}
${ }^{1}$ Department of Obstetrics and Gynecology, University Hospitals Plymouth NHS Trust, Plymouth, United Kingdom ${ }^{2}$ Department of Obstetrics and Gynecology, Bombay Hospital and Medical Research Centre, Mumbai, Maharashtra, India
\end{abstract}

Received: 03 February 2020

Accepted: 11 February 2020

\author{
*Correspondence: \\ Dr. Naimah Raza, \\ E-mail: naimahr91@gmail.com
}

Copyright: ( $)$ the author(s), publisher and licensee Medip Academy. This is an open-access article distributed under the terms of the Creative Commons Attribution Non-Commercial License, which permits unrestricted non-commercial use, distribution, and reproduction in any medium, provided the original work is properly cited.

\begin{abstract}
Background: Dengue is one of the most important mosquito-borne diseases in India. This study was done to study the course of dengue infection in pregnant women, its effects, foeto-maternal outcome, and associated complications. Methods: Clinical, laboratory findings, and maternal/foetal outcome were collected from patients with confirmed dengue infections in pregnancy treated at a tertiary care hospital in India, during a 6-month period.

Results: A total 12 seropositive cases of dengue infection in pregnancy were diagnosed during the study period. Thrombocytopenia coupled with rising haematocrit were seen in all cases. Prophylactic platelet transfusion was not necessary in any of the cases without bleeding manifestation. Three patients developed severe dengue with some complication requiring extra measures, including a case of dengue encephalitis which responded to supportive treatment. One case of intra-uterine foetal demise and multi-organ failure leading to maternal death was seen. Perinatal outcomes of the other cases were satisfactory.

Conclusions: Dengue in pregnancy requires early diagnosis and treatment. A high index of clinical suspicion is essential in any pregnant woman with fever, especially in endemic areas. Further studies are necessary as evidencebased data in the management of dengue in pregnancy is inadequate.
\end{abstract}

Keywords: Dengue, Fever, Pregnancy, Thrombocytopenia

\section{INTRODUCTION}

Dengue infection is a mosquito-borne febrile illness occurring in tropical and subtropical areas. Dengue is one of the most important mosquito-borne diseases in India and epidemics have become more common. Based on the data of National Vector Borne Disease Control Programme (NVBDCP), the number of cases reported in 2017 was about 188,401 for dengue with 325 deaths. ${ }^{1}$ It is caused by four closely related dengue virus serotypes (designated DENV-1, DENV-2, DENV-3, and DENV-4) of the genus Flavivirus, family Flaviviridae. The various serotypes of the dengue virus are transmitted to humans through the bites of infected Aedes mosquitoes, principally Ae. aegypti. (Others include Ae. albopictus, Ae. polynesiensis, and Ae. scutellaris complex).

The 2009 WHO criteria classify dengue according to levels of severity: dengue without warning signs; dengue with warning signs (abdominal pain, persistent vomiting, fluid accumulation, mucosal bleeding, lethargy, liver enlargement, increasing haematocrit with decreasing platelets); and severe dengue (dengue with severe plasma leakage, severe bleeding, or organ failure). ${ }^{2}$

Pregnancy does not appear to increase the incidence or severity of dengue infection. ${ }^{3}$ However, an infection in pregnancy carries the risk of haemorrhage for both the 
mother and the new-born. There is also a risk of premature birth, vertical transmission causing neonatal thrombocytopenia, and foetal death. ${ }^{4,5}$

Laboratory diagnostic methods for confirming dengue infection may involve detection of the virus, viral nucleic acid, antigens or antibodies, or a combination of these techniques.

\section{METHODS}

All serologically diagnosed pregnant women treated for dengue infection during a 6-month period (June to November, 2017) at a tertiary care hospital in India were included.

\section{Inclusion criteria}

- All serologically diagnosed pregnant women (antenatal, intra-partum, and immediate post-partum) diagnosed with dengue infection were included in the study

- Rapid NS-1 antigen detection test and serological test for detection of antibodies was used to confirm the diagnosis. Serological diagnosis depends on the presence of $\mathrm{IgM}$ antibody or a rise in $\mathrm{IgG}$ antibody titre in paired acute and convalescent phase serum.

\section{Exclusion criteria}

- Patients with febrile illness other than dengue infection, and with pre-existing medical disorders including hematological disorders, were excluded.

Clinical data, laboratory findings, and maternal and foetal outcomes were documented prospectively during their hospital stay. The cases were followed up daily for their clinical and laboratory parameters. Laboratory parameters that were followed up included a complete hemogram with haematocrit, total leucocyte count, and manual platelet count. Extra investigations (liver function tests, renal function tests etc.) were done whenever deemed necessary.

Grading of the severity of dengue infections was done according to 2009 World Health Organization (WHO) criteria. ${ }^{2}$ Cases were classified as:

- Dengue without warning signs

- Dengue with warning signs (abdominal pain, persistent vomiting, fluid accumulation, mucosal bleeding, lethargy, liver enlargement, increasing haematocrit with decreasing platelets); and

- Severe dengue (dengue with severe plasma leakage, severe bleeding, or organ impairment).

\section{Analysis}

Cases were evaluated based on the fall in platelet count, rise in haematocrit, presence of haemorrhagic manifestations or fluid leak in the mother, need for admission to an intensive care unit, need for platelet transfusion, the mode of delivery and the foeto-maternal outcome. The results were obtained and tabulated in an excel sheet. Data was then analysed manually.

The study protocol was approved by the Institutional Review Board. An informed consent was obtained from all the patients included in the study.

\section{RESULTS}

Twelve pregnant women were diagnosed with dengue infection during the study period. Their clinical and laboratory findings are shown in Table 1.

Two patients $(16.66 \%)$ presented in the first trimester of pregnancy, three $(25 \%)$ in the second, six $(50 \%)$ in the third trimester, while one $(8.33 \%)$ presented in immediate post-partum period.

Three women (25\%) developed severe dengue, while the remaining nine $(75 \%)$ belonged to the category of Dengue with presence of warning signs. Thrombocytopenia, coupled with raised haematocrit were seen in all cases.

Conservative management in the form of hydration, antipyretic drugs, supportive care, and close monitoring of clinical condition and laboratory findings were sufficient in all cases of uncomplicated dengue infection. There was no place for prophylactic platelet transfusion, even in cases with severe thrombocytopenia. Platelet transfusion was needed only in cases with bleeding manifestations ( 3 out of 12 cases i.e. $25 \%)$. Three patients $(25 \%)$ needed admission to an intensive care unit for management. A total 9 out of the total $12(75 \%)$ cases were managed without any complications. The most common presenting complaints were fever and myalgia. Maternal and foetal outcomes were satisfactory in all of these cases.

Three cases $(25 \%)$ presented with severe dengue, and developed complications in the form of ARDS causing death, bleeding, and encephalitis respectively. A few details about these three cases are mentioned below.

Case \#10 was a 25-year-old woman, G2P1L1, 23 weeks gestation, referred from another hospital in a critical and intubated state. Patient was hospitalised earlier with complaints of fever with chills, and was diagnosed with dengue infection (platelet count - 30,000/cumm). She developed breathlessness at the hospital, with ultrasound of thorax suggestive of left lung consolidation. She deteriorated over the day, needed intubation, and was transferred to our centre for specialized intensive care management. Patient was received in a compromised state. (Pulse - 130/min; BP - 100/60 mmHg; $\mathrm{SpO}_{2}-57 \%$ ). She was given 8 units of platelet and 2 units of packed cell transfusion. Ultrasound scan was suggestive of intrauterine foetal demise. Induction of labour was done with 
vaginal misoprostol tablets and dead foetus was expelled a few hours later, with no active vaginal bleeding. She required inotropic support in view of falling pressures and decreased urine output. Chest X-ray was suggestive of acute respiratory distress syndrome (ARDS) and cardiomegaly. Increased inotropic support failed to maintain the blood pressures. Her arterial blood gas evaluation showed respiratory and metabolic acidosis. Patient further developed bradycardia and then cardiac arrest. Despite all resuscitative measures, the patient could not be revived, and was declared dead.

Table 1: Clinical and laboratory characteristics with foeto-maternal outcomes.

\begin{tabular}{|c|c|c|c|c|c|c|c|c|c|c|c|}
\hline 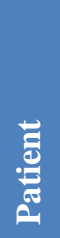 & 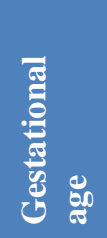 & 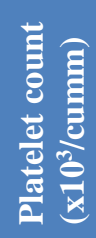 & 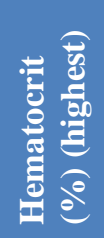 & 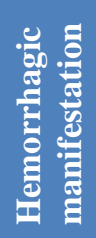 & 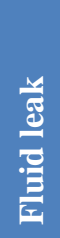 & 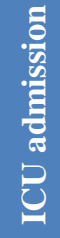 & 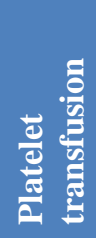 & 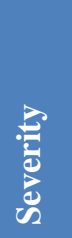 & 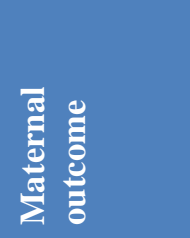 & 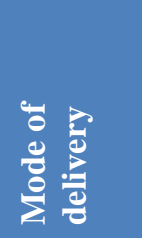 & 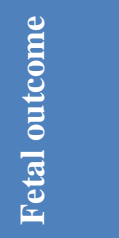 \\
\hline 1. & 11 & 47 & 46 & $\mathrm{~N}$ & $\mathrm{~N}$ & $\mathrm{~N}$ & $\mathrm{~N}$ & DW & Normal & FTNVD & Normal \\
\hline 2. & 18 & 48 & 49 & $\mathrm{~N}$ & $\mathrm{~N}$ & $\mathrm{~N}$ & $\mathrm{~N}$ & DW & Normal & FTNVD & Normal \\
\hline 3. & 31 & 86 & 44 & $\mathrm{~N}$ & $\mathrm{~N}$ & $\mathrm{~N}$ & $\mathrm{~N}$ & DW & Normal & FTNVD & Normal \\
\hline 4. & 36 & 22 & 47 & $\mathrm{~N}$ & $\mathrm{~N}$ & $\mathrm{~N}$ & $\mathrm{~N}$ & DW & Normal & FTNVD & Normal \\
\hline 5. & 35 & 39 & 46 & $\mathrm{~N}$ & $\mathrm{~N}$ & $\mathrm{~N}$ & $\mathrm{~N}$ & DW & Normal & LSCS* & Normal \\
\hline 6. & 9 & 40 & 45 & $\mathrm{~N}$ & $\mathrm{~N}$ & $\mathrm{~N}$ & $\mathrm{~N}$ & DW & Normal & FTNVD & Normal \\
\hline 7. & 27 & 32 & 42 & $\mathrm{~N}$ & $\mathrm{~N}$ & $\mathrm{~N}$ & $\mathrm{~N}$ & DW & Normal & FTNVD & Normal \\
\hline 8. & 37 & 56 & 44 & $\mathrm{~N}$ & $\mathrm{~N}$ & $\mathrm{~N}$ & $\mathrm{~N}$ & DW & Normal & FTNVD & Normal \\
\hline 9. & 36 & 85 & 46 & $\mathrm{~N}$ & $\mathrm{~N}$ & $\mathrm{~N}$ & $\mathrm{~N}$ & DW & Normal & FTNVD & Normal \\
\hline 10. & 23 & 30 & 46 & $Y$ & $Y$ & $Y$ & Y & SD & Death & VD & IUFD \\
\hline 11. & 38 & 49 & 42 & $\mathrm{Y}$ & $\mathrm{N}$ & $Y$ & Y & SD & Bleeding & FTNVD & Normal \\
\hline 12. & 39 & 14 & 49 & $\mathrm{Y}$ & $\mathrm{Y}$ & $\mathrm{Y}$ & $\mathrm{Y}$ & SD & Encephalitis & LSCS** & Normal \\
\hline
\end{tabular}

Y: Yes, N: No, DW: Dengue with warning signs, SD: Severe dengue, FTNVD: Full term normal vaginal delivery, LSCS: Lower segment caesarean section, IUFD: Intra uterine foetal death, *LSCS due to non-progress of labour at 39 weeks, after recovery from dengue infection, **LSCS due to second stage arrest with meconium stained liquor. Pre-operative platelet count- 67,000/cumm.

Case \#11 was a 38-year-old woman, G2A1, 38 weeks gestation, a known case of deep vein thrombosis (on low molecular weight heparin). She came in active labour and underwent a vaginal delivery with a left mediolateral episiotomy (male baby $2.5 \mathrm{~kg}$ ). While the patient gave no history of fever prior to admission, on day 3 postdelivery, she developed high grade fever associated with chills and body ache. Investigations confirmed a dengue infection, with a platelet count of 49,000/cumm. The next day, she developed swelling at the episiotomy site, which was tense, tender and pitting on examination, suggestive of a hematoma with increasing size. Anticoagulation was stopped. The patient was visibly pale with a haemoglobin of $5.7 \mathrm{gm} \%$. She was posted for drainage in the operation theatre for hematoma evacuation. Post-surgery, she was shifted to ICU for close monitoring of vitals. She received 5 units of packed cells, and 12 units of platelet transfusion. Once bleeding stopped completely and patient stabilized, she was shifted out of the ICU. Drainage site was left to be healed with secondary intention. Patient was discharged on $14^{\text {th }}$ day postdelivery. She was followed up later regarding the need for re-starting thromboprophylaxis.
Case \#12 was a 30-year-old woman, G3P2L2, 39 weeks gestation, transferred from a secondary centre in view of dengue fever with thrombocytopenia (platelet count67,000). She underwent an emergency caesarean in view of second stage arrest with meconium stained liquor. There was excessive intra-operative bleeding, for which a bilateral internal iliac artery ligation had to be done. In the ICU, on day 1 post-surgery, she had an episode of generalized tonic clonic convulsion. Neurology opinion was sought, and patient was started on injection Levetiracetam. The patient was conscious, but violent and non-cooperative. MRI was suggestive of features of encephalitis. Patient received 5 units of packed cell and 16 units FFPs in total. She was monitored in the ICU, on intra-venous antibiotics, steroids and supportive care. Her neurological status gradually improved and as she stabilized, she was shifted out of ICU.

Foetal outcomes were satisfactory in all but one of the pregnancies $(8.33 \%)$, which had foetal demise. There were no cases suggestive of vertical transmission causing anomalies or need for platelet transfusions in the neonate due to bleeding manifestations. Routine screening using dengue-specific IgM antibodies in cord blood or serum 
for vertical transmission was not performed due to financial constraints.

All the women and new-borns who were discharged from the hospital were reviewed in postnatal clinics and paediatric clinics. None of the babies showed clinical evidence of ill health (including bleeding manifestations) or failure to thrive.

\section{DISCUSSION}

Dengue is a major public health problem in tropical countries. We encountered 12 cases of seropositive dengue infection in pregnancy during our study period at a tertiary hospital in India. There are several reports of dengue infection in pregnancy from literature review (Table 2).

Table 2: Summarized literature of cases of dengue infection in pregnancy (since 2006).

\begin{tabular}{|c|c|c|c|}
\hline Authors & Country & Maternal outcome & Fetal outcome \\
\hline Waduge et $\mathrm{al}^{6}$ & Sri Lanka & $\begin{array}{l}\text { Thrombocytopenia, pleural effusion, } \\
\text { hepatomegaly, myocarditis }\end{array}$ & Low birth weight, miscarriage \\
\hline Leon $\mathrm{R}$ et $\mathrm{al}^{7}$ & Mexico & $\begin{array}{l}\text { Premature labour, pleural effusion, } \\
\text { oligohydramnios, postpartum hemorrhage }\end{array}$ & Neonatal sepsis \\
\hline Phongsamart et $\mathrm{al}^{8}$ & Thailand & Thrombocytopenia, rash & Fever, petechiae, hepatomegaly \\
\hline Singh et $\mathrm{al}^{9}$ & India & Post-partum hemorrhage & $\begin{array}{l}\text { Fetal distress during labor, } \\
\text { erythematous rash, hepatosplenomega }\end{array}$ \\
\hline Basurko et al ${ }^{10}$ & $\begin{array}{l}\text { French } \\
\text { Guiana }\end{array}$ & $\begin{array}{l}\text { Premature labour, hemorrhage during } \\
\text { labour, abruptio placentae }\end{array}$ & $\begin{array}{l}\text { Prematurity, intrauterine fetal death, } \\
\text { late miscarriage, acute fetal distress } \\
\text { during labor, neonatal death }\end{array}$ \\
\hline Adam I et al ${ }^{11}$ & $\begin{array}{l}\text { Eastern } \\
\text { Sudan }\end{array}$ & Death, preterm delivery & Low birth weight, neonatal death \\
\hline Chitra TV et al ${ }^{12}$ & India & Severe thrombocytopenia, bleeding & None \\
\hline Agrawal et al ${ }^{13}$ & India & $\begin{array}{l}\text { Miscarriage, premature labour, ante- } \\
\text { partum and post-partum hemorrhage, } \\
\text { oligohydramnios }\end{array}$ & Intra-uterine death, neonatal death \\
\hline
\end{tabular}

The severity of the clinical picture varies as evident in Table 2. Symptoms of infected women often differ. In general, the most common symptoms include fever, myalgia and arthralgia. $^{14}$ Fluid leakage (elevated haematocrit, pleural effusion or ascites) and hemorrhagic manifestations are characteristic features of dengue hemorrhagic fever, however, the physiological hemodilution of normal pregnancy can mask the hemoconcentration in dengue. ${ }^{6}$

It is well proven that sequential infection with different dengue serotypes predisposes to more severe forms of the disease. This is explained by enhancement of the crossreactive cascade of amplified non-neutralizing heterologous antibodies, cytokines (higher levels of TNF$\alpha$, IFN- $\gamma$ ) and complement activation causing endothelial dysfunction, platelet destruction and consumptive coagulopathy. ${ }^{15-17}$

Adverse foetal outcomes may be attributed to the effects on placental circulation caused by endothelial damage with increased vascular permeability leading to plasma leakage. ${ }^{5,15}$

In our study of 12 cases, we observed maternal complications like thrombocytopenia, bleeding, encephalitis, and acute respiratory distress syndrome causing maternal death. Foetal outcomes were satisfactory in all but one case, which had intra-uterine foetal demise.

The study is limited by the diagnostic tests that were used for detecting dengue infection. The widely available clinical diagnostic test (the method used for the study) for investigating dengue is the rapid strip test with a sensitivity and specificity of $\approx 90 \%$. The capture ELISA tests are comparatively more sensitive and specific $(\approx$ $95 \%$ and $100 \%$ respectively). Therefore, our study might have underestimated the actual outcomes. None of the kits mentioned above has the ability to differentiate between the serotypes. On the other hand, the gold standard, reverse transcriptase PCR using type-specific primers is highly sensitive and specific. It is only positive during the acute phase and becomes negative shortly after defervescence, so the detection window in the clinical setup to confirm infection is relatively narrow. ${ }^{18}$

\section{CONCLUSION}

Dengue in pregnancy requires early diagnosis and treatment. Early diagnosis is made difficult by the ambiguity of clinical findings and physiological changes of pregnancy that may confuse the clinician. A high index of clinical suspicion is, therefore, essential in any 
pregnant female with fever during epidemics. Pregnancies complicated by dengue infection require close monitoring for potential maternal and foetal complications. Conservative medical and obstetric management is the treatment of choice. Platelet transfusion is needed only in cases complicated by haemorrhagic manifestations.

Further studies and systematic reviews are mandatory as evidence-based data in the management specific for pregnant patients are inadequate.

\section{ACKNOWLEDGMENTS}

Authors would like to thank the institute, Bombay Hospital and Medical Research Centre, for allowing us to conduct our study.

\section{Funding: No funding sources}

Conflict of interest: None declared

Ethical approval: The study was approved by the Institutional Ethics Committee

\section{REFERENCES}

1. Dengue/DHF situation in India: National Vector Borne Disease Control Programme (NVBDCP). Nvbdcp.gov.in. Available at: https://nvbdcp.gov.in/index4.php?lang=1\&level=0\&1 inkid $=431 \&$ lid $=3715$. Accessed on $6^{\text {th }}$ February 2020.

2. World Health Organization. Dengue: Guidelines for diagnosis, treatment, prevention and control. Geneva, Switzerland: World Health Organization; 2009. Available at: http://apps.who.int/iris/bitstream/10665/44188/1/978 9241547871_eng.pdf. Accessed on $6^{\text {th }}$ February 2020.

3. Carroll ID, Toovey S, Van Gompel A. Dengue fever and pregnancy: a review and comment. Travel Med Infect Dis. 2007;5(3):183-8.

4. Chotigeat U, Kalayanarooj S, Nisalak A. Vertical transmission of dengue infection in Thai infants: two case reports. J Med Assoc Thai. 2003;86(SUPP/3):628-32.

5. Chye JK, Lim CT, Ng KB, Lim JM, George R, Lam SK. Vertical transmission of dengue. Clin Infect Dis. 1997;25(6):1374-7.

6. Waduge R, Malavige GN, Pradeepan M, Wijeyaratne CN, Fernando S, Seneviratne SL. Dengue infections during pregnancy: a case series from Sri Lanka and review of the literature. J Clin Virol. 2006;37:27-33.

7. Rosado Leon R, Munoz Rodriguez MR, Soler Huerta E, Parissi Crivelli A, Mendez Machado GF.
Dengue fever during pregnancy. Cases Report. Ginecol Obstet Mex. 2007;75:687-90.

8. Phongsamart W, Yoksan S, Vanaprapa N, Chokephaibulkit K. Dengue virus infection in late pregnancy and transmission to the infants. Pediatr Infect Dis J. 2008;27:500-4.

9. Singh N, Sharma KA, Dadhwal V, Mittal S, Selvi AS. A successful management of dengue fever in pregnancy: report of two cases. Indian $\mathrm{J}$ Med Microbiol. 2008;26:377-80.

10. Basurko C, Carles G, Youssef M, Guindi WE. Maternal and foetal consequences of dengue fever during pregnancy. Eur J Obstet Gynecol Reprod Biol. 2008;147:29-32.

11. Adam I, Jumaa AM, Elbashir HM, Karsany MS. Maternal and perinatal outcomes of dengue in Port Sudan, Eastern Sudan. Virol J. 2010;7(1):153.

12. Chitra TV, Panicker S. Maternal and foetal outcome of dengue fever in pregnancy. J Vector Borne Dis. 2011;48(4):210.

13. Agrawal P, Garg R, Srivastava S, Verma U, Rani R. Pregnancy outcome in women with dengue infection in Northern India. Indian J Clin Pract. 2014;24(11):1053-6.

14. Malavige GN, Velathanthiri VG, Wijewickrama ES, Fernando S, Jayaratne SD, Aaskov J, et al. Patterns of disease among adults hospitalized with dengue infections. QJM. 2006;99:299-305.

15. Iyngkaran N, Yadav M, Sinniah M. Augmented inflammatory cytokines in primary dengue infection progressing to shock. Singapore Med J. 1995;36:218-21.

16. Soundravally R, Hoti SL, Patil SA, Cleetus CC, Zachariah B, Kadhiravan $\mathrm{T}$, et al. Association between proinflammatory cytokines and lipid peroxidation in patients with severe dengue disease around defervescence. Int J Infect Dis. 2014;18:6872.

17. Halstead SB. Dengue antibody-dependent enhancement: knowns and unknowns. Microbiol Spectr. 2014;2(6):249-71.

18. Sa-ngasang A, Wibulwattanakij S, Chanama S, Orapinpatipat A, Anuegoonpipat A, Anantapreecha $\mathrm{S}$, et al. Evaluation of RT-PCR as a tool for diagnosis of secondary dengue virus infection. Jpn J Infect Dis. 2003;56:205-9.

Cite this article as: Raza N, Dhungat NP. Outcome of Dengue infection in pregnancy at a tertiary care hospital in India. Int J Reprod Contracept Obstet Gynecol 2020;9:970-4. 